\title{
Design Cost and Spectrum Efficiency Comparison of Fixed-Grid and Flex-Grid Optical Networks with Grooming
}

\author{
Amaro de Sousa \\ Instituto de Telecomunicações \\ Universidade de Aveiro \\ Aveiro, Portugal \\ asou@ua.pt
}

\author{
Carlos Borges Lopes \\ Instituto de Telecomunicações \\ Aveiro, Portugal \\ clopes@av.it.pt
}

\author{
Paulo Monteiro \\ Instituto de Telecomunicações \\ Universidade de Aveiro \\ Aveiro, Portugal \\ paulo.monteiro@ua.pt
}

\begin{abstract}
This paper deals with the design of optical networks aiming to minimize the design cost and the spectrum usage. We present an optimization method that is able to find the optimal solutions of relevant sized instances. We apply the method to realistic case studies. With the obtained solutions, we make a cost and spectrum efficiency comparison analysis between fixed-grid and flex-grid optical networks. The results show that flex-grid gains are significant over the fixed-grid alternatives only after the introduction of $400 \mathrm{Gbps}$ line rates and marginal gains are obtained without this line rate.
\end{abstract}

Keywords - fixed-grid, flex-grid, optical network design, optimization

\section{INTRODUCTION}

Fixed-grid optical networks, which are based on the 50 $\mathrm{GHz}$ spectrum grid, are not adequate to support the future 400 Gbps line rates. On the other hand, flex-grid allows for finer spectrum granularities at the expense of increased complexity and capital investment. Thus, the comparison of cost and spectrum efficiency between fixed-grid and flex-grid is an important issue for network operators that need to know when to evolve their networks.

This paper deals with the design of optical networks aiming to minimize the design cost and the spectrum usage. The problem addressed here includes the grooming of client demands over the lightpaths, the routing of the lightpaths over the fiber network and the spectrum assignment of each lightpath. With flex-grid used in elastic networks, different modulation formats for the same line rate exhibit different tradeoffs between optical transparent reach (which has a direct impact on the required number of regenerators) and spectrum usage. In this work, we assume end-to-end grooming, i.e., client demands can be groomed only by a direct lightpath between their end nodes. We study this problem in the context of core networks that, due to fiber lengths, may require the use of electrical regenerators. Nevertheless, we impose that regenerators must be placed only on intermediate nodes (i.e., no electrical regeneration can by placed in the middle of a fiber connection).
The paper is organized as follows. In section II, we briefly describe some related work. In section III, we present the optimization method that is able to find the optimal solutions of relevant sized instances. In section IV, we present the computational results obtained by the optimization method in problem instances based on the NSFNET network topology and, with the obtained solutions, we make a cost and spectrum efficiency comparison analysis between fixed-grid and flexgrid optical networks. Finally, in section V, we draw the main conclusions of this work.

\section{RELATED WORK}

Recently, it has been shown in [1] that Orthogonal Frequency-Division Multiplexing (OFDM) transponders have significant spectrum benefits over typical fixed-grid WDM networks. A comparison between fixed-grid network architectures and variable-spacing OFDM solutions shows that capacity gains can reach up to $50 \%$ but are strongly affected by physical and topological constraints of transparent networks and traffic statistics [2].

Cost comparisons between mixed line rate and elastic networks show that elastic networks can bring up to $37 \%$ lower cost, particularly for high loads and dynamic scenarios [3]. On the other hand, in [4], spectrum and energy efficiency comparisons between fixed-grid and flex-grid networks show that the traffic load has a great impact on the network costs. In [5], the network planning is formulated as a multi-objective optimization problem with respect to the maximum spectrum and the cost of transponders used and presents Integer Linear Programming (ILP) models and heuristic algorithms to solve it. In [6], a similar problem is tacked only by heuristic methods. On both cases, the heuristic methods are applied to flex-grid OFDM networks and mixed line rate fixed-grid WDM networks and the results of the heuristic algorithms are compared for these cases.

In those works, though, the comparisons were conducted through heuristic method results that can have some errors associated to the non-optimality of the methods. In here, cost and spectrum efficiency comparisons are based on optimal solutions provided by an exact method. Other recent works 
address related issues, such as adequate support of dynamic traffic and spectrum fragmentation (in [7]) and the tradeoff between network cost and problem complexity according to traffic grooming, regeneration and modulation assignment in the routing and spectrum allocation problem (in [8]).

\section{OPTIMIZATION METHOD}

The complete problem is solved in two steps. In the first step, we solve the grooming problem aiming to minimize the network cost. In the second step, we solve the routing and spectrum assignment problem, guaranteeing the previous computed minimum cost and aiming to minimize the highest assigned spectrum slot. Note that the highest assigned slot gives us a measure on how much total spectrum is required to support a given demand set with a minimum cost and this value is used to compare this parameter among different design solutions.

The proposed two-step approach is exact under the mild condition that the solution obtained in the first step is feasible in the second step problem. This condition requires the total required client demand not being too close to the total capacity of the network. This is, though, the usual operating condition of real networks since network operators usually upgrade their network capacities significantly before their full use.

To simplify the notation, we use the term 'spectrum slots' indiscriminately for fixed-grid and flex-grid networks. These spectrum slots should be interpreted as $50 \mathrm{GHz}$ channels in fixed-grid (in this case, each lightpath is assigned with one spectrum slot) and, in our case, as $25 \mathrm{GHz}$ slots in flex-grid (each lightpath can be assigned more than one slot as long as they are contiguous). In the following, we describe each step separately.

\section{A. First Step}

For a given set of client demands, the aim is to compute a minimum cost set of lightpaths that must be established on the fiber network. We consider that client demands are of Ethernet type and can be of $10 \mathrm{Gbps}, 40 \mathrm{Gbps}$ or $100 \mathrm{Gbps}$. In addition, we assume that lightpaths can be of 3 types: OTU3 (with a line rate of $40 \mathrm{Gbps}$ ), OTU4 (with a line rate of $100 \mathrm{Gbps}$ ) and OTU5 (with a line rate of $400 \mathrm{Gbps).} \mathrm{Each} \mathrm{lightpath} \mathrm{is} \mathrm{defined}$ by a source node, a target node, an OTU type (that defines its line rate), a modulation format (that defines the number of required contiguous slots and the maximum reach) and the number of required regenerators.

Following [9], we assume that the following transport alternatives exist:

- Client demands of 10 Gbps Ethernet type can be transported in OTU3 lightpaths (each one supporting 4 demands) or in OTU4 lightpaths (each one supporting 10 demands).

- Client demands of 40 Gbps Ethernet type can be transported in OTU3 lightpaths (each one supporting 1 demand) or in OTU4 lightpaths (each one supporting 2 demands) or in OTU5 lightpaths (each one supporting 10 demands).
- Client demands of 100 Gbps Ethernet type can be transported in OTU4 lightpaths (each one supporting 1 demand) or in OTU5 lightpaths (each one supporting 4 demands).

In this case, and since we consider end-to-end grooming, the grooming problem can be addressed separately for each source-target pair of nodes and each type of client demand between these nodes. For each source-target node pair and each client demand type, we solve an Integer Linear Programming (ILP) model to compute the set of minimum cost required lightpaths.

Let us consider one illustrative example. Consider a sourcetarget pair of nodes requiring $d$ demands of $10 \mathrm{Gbps}$ Ethernet type. As described before, these demands can be groomed in lightpaths of type OTU3 or type OTU4. In this example, the appropriate ILP model is:

$$
\begin{aligned}
& \text { Minimize } m=c_{x} x+c_{y} y \\
& \text { Subject to } \\
& 4 x+10 y \geq d \\
& x \text { and } y \text { integers }
\end{aligned}
$$

In this ILP model, $c_{x}$ is the minimum cost of deploying a lightpath of type OTU3 and $c_{y}$ is the minimum cost of deploying a lightpath of OTU4. These cost parameters are determined in advance as the minimum cost among all modulation formats available for the respective OTU types and are given by the sum of the costs of the muxponders/ transponders and the cost of the minimum number of regenerators required between the source and target nodes of the demand set. When solving this ILP model, the values of the variables $x$ and $y$ are, respectively, the number of required lightpaths of OTU3 and OTU4 of the optimal solution. Note that constraint (2) guarantees that each lightpath of type OTU3 supports up to $410 \mathrm{Gbps}$ client demands while each lightpath of type OTU4 supports up to $1010 \mathrm{Gps}$ client demands (as previously described) - for different demand types and different available OTU types, constraint (2) is defined accordingly. The number of OTU3 and OTU4 lightpaths determined by variables $x$ and $y$ are added to the set of lightpaths to be established in the network in the second step. The total cost of the solution is the sum of all $m$ values, where each $m$ value is obtained by solving a similar ILP for each source-target node pair and each client demand type.

\section{B. Second Step}

In the second step, we solve the routing and spectrum assignment problem with a single compact ILP model aiming to minimize the highest assigned spectrum slot. For each lightpath, determined in the first step, we jointly select a routing path and assign a contiguous set of spectrum slots on each link belonging to the path. The routing path is composed by a concatenation of subpaths that are limited in length by the modulation format reach of the lightpath. Between the end of a subpath and the beginning of the next subpath, a regenerator is needed. The ILP model has constraints: (1) to define a routing path for each ligthpath from its source node to its target node, 
(2) to assign spectrum slots for each subpath, (3) to guarantee the spectrum continuity along the subpaths, (4) to limit the length of the subpaths to the modulation format reach, (5) to guarantee that each spectrum slot on each fiber is assigned to at most one lightpath and (6) to allow for spectrum slot conversion between the subpaths of each routing path. Also, some additional constraints were developed to reduce the ILP solving time.

\section{RESUlts}

In our computational results, we have considered the 14node NSFNET network [10] with the fiber lengths, in Km, presented in Fig. 1. In all cases, we have considered client Ethernet types of 10, 40 and $100 \mathrm{Gbps}$. For each type, a set of $n$ source-target pairs of nodes were randomly selected and, for each pair of nodes, the number of client demands $d$ was generated with a uniform distribution between $d_{\min }$ and $d_{\max }$. Table I shows the 6 different demand combinations $(20 \mathrm{~L}, 20 \mathrm{H}$, $30 \mathrm{~L}, 30 \mathrm{H}, 40 \mathrm{~L}, 40 \mathrm{H})$ considered in the computational results. For each demand combination, the traffic demand parameters of each demand type are presented in the form $n \times\left[d_{\min } ; d_{\max }\right]$. For each combination, two case studies were randomly generated (for example, 20L1 and 20L2 represent the two case studies randomly generated for the 20L combination).

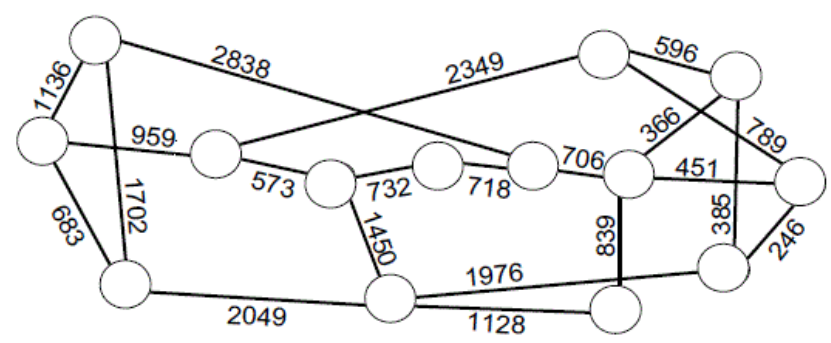

Fig. 1. NSFNET network topology

In all case studies, we have considered a spectrum capacity of $4 \mathrm{THz}$ on each optical link representing a capacity of 80 slots of $50 \mathrm{GHz}$, in the fixed-grid scenarios, and of 160 slots of $25 \mathrm{GHz}$, in the flex-grid scenarios. Table II presents, for each client demand type, the OTU alternatives together with their characterization in terms of spectrum usage in $\mathrm{GHz}$ (' $\mathrm{S}$ '), muxponder/transponder cost ('trCost'), the cost of each required regenerator ('rCost') and the transparent reach ('reach'), in Km, as proposed in the cost models presented in [9]. Note that, in fixed-grid, we have considered only the alternatives whose spectrum usage is $50 \mathrm{GHz}$ (the others correspond to flex-grid alternatives).

TABLE I. TRAFFIC DEMAND PARAMETERS

\begin{tabular}{|c|c|c|c|}
\hline & 10 Gbps & 40 Gbps & 100 Gbps \\
\hline $20 \mathrm{~L}$ & $20 \times[4 ; 16]$ & $12 \times[2 ; 8]$ & $4 \times[1 ; 4]$ \\
\hline $20 \mathrm{H}$ & $20 \times[6 ; 24]$ & $12 \times[3 ; 12]$ & $4 \times[1 ; 6]$ \\
\hline $30 \mathrm{~L}$ & $30 \times[4 ; 16]$ & $18 \times[2 ; 8]$ & $6 \times[1 ; 4]$ \\
\hline $30 \mathrm{H}$ & $30 \times[6 ; 24]$ & $18 \times[3 ; 12]$ & $6 \times[1 ; 6]$ \\
\hline $40 \mathrm{~L}$ & $40 \times[4 ; 16]$ & $24 \times[2 ; 8]$ & $8 \times[1 ; 4]$ \\
\hline $40 \mathrm{H}$ & $40 \times[6 ; 24]$ & $24 \times[3 ; 12]$ & $8 \times[1 ; 6]$ \\
\hline
\end{tabular}

TABLE II. LIGHTPATH COST MODEL

\begin{tabular}{|c|c|c|r|r|r|}
\hline Demand & OTU & \multicolumn{1}{c|}{ S } & trCost & rCost & reach \\
\hline \multirow{3}{*}{ 10Gbps } & OTU3 & 50 & 5 & 9.6 & 2500 \\
\cline { 2 - 6 } & OTU4 & 50 & 13 & 24 & 2000 \\
\cline { 2 - 6 } & OTU4 & 75 & 16 & 35.2 & 2500 \\
\hline \multirow{4}{*}{ 40Gbps } & OTU3 & 50 & 6 & 9.6 & 2500 \\
\cline { 2 - 6 } & OTU4 & 50 & 16 & 24 & 2000 \\
\cline { 2 - 6 } & OTU4 & 75 & 19 & 35.2 & 2500 \\
\cline { 2 - 6 } & OTU5 & 100 & 18 & 24 & 1000 \\
\hline \multirow{3}{*}{ 100Gbps } & OTU4 & 50 & 15 & 24 & 2000 \\
\cline { 2 - 6 } & OTU4 & 75 & 22 & 35.2 & 2500 \\
\cline { 2 - 6 } & OTU5 & 100 & 16 & 24 & 1000 \\
\hline
\end{tabular}

We have considered 3 scenarios: (A) a fixed-grid scenario with only OTU3 and OTU4 fixed grid alternatives for the lightpaths, (B) a flex-grid scenario with OTU3 and OTU4 fixed-grid and flex-grid alternatives for the lightpaths and (C) a flex-grid scenario with all alternatives (i.e., including the OTU5 flex-grid alternatives).

The computational results were obtained using CPLEX 12.4 in a PC with a Core i7 CPU, 4 GB of RAM and Windows 7 OS. The first step which consists on solving the small ILPs for all source-target node pairs and all client demand types is solved within a few seconds in total. The second step took from a few minutes (in the fixed-grid scenarios) to some hours (in the flex-grid scenarios). In all cases, though, we have obtained the provable optimal solutions. The 12 case studies represent a total supported client demand from 5.4 Tbps (case 20L2) up to $16.5 \mathrm{Tbps}$ (case $40 \mathrm{H} 2$ ).

Table III presents the cost and spectrum usage of the obtained optimal solutions of fixed-grid and flex-grid scenarios. Column 'CostA' and 'SpecA' show respectively the cost and the number of $25 \mathrm{GHz}$ slots of used spectrum obtained for the fixed-grid scenario A (as the granularity of slots considered in fixed-grid is $50 \mathrm{GHz}$, in this scenario this number is always even). Columns 'CostB' and 'SpecB' show respectively the cost and the number of $25 \mathrm{GHz}$ slots of spectrum for flex-grid solutions without $400 \mathrm{Gbps}$ line rates (scenario B); columns 'CostC' and 'SpecC' show the same results for flex-grid solutions with $400 \mathrm{Gbps}$ line rates (scenario C). The optimal solutions of fixed-grid scenarios exhibit a spectrum usage between 30 slots of $50 \mathrm{GHz}$ (case 20L1) and 77 slots of $50 \mathrm{GHz}$ (case 40H1). The optimal solutions of flex-grid scenarios without $400 \mathrm{Gbps}$ line rates exhibit a spectrum usage between 54 slots of $25 \mathrm{GHz}$ (case 20L1) and 154 slots of $25 \mathrm{GHz}$ (case 40H1); and, for flex-grid scenarios with $400 \mathrm{Gbps}$ line rates, the spectrum usage is between 52 slots of $25 \mathrm{GHz}$ (case 20L1) and 128 slots of 25 $\mathrm{GHz}$ (case 40H2).

The cost and spectrum usage comparisons between the obtained optimal solutions of fixed-grid and flex-grid scenarios are presented in Table IV. This table presents the cost gain (column 'Cost1') and spectrum usage gain (column 'Spec1') of flex-grid solutions without $400 \mathrm{Gbps}$ line rates (scenario B) when compared with fixed-grid solutions (scenarios A). This 
table also presents the cost gain (column 'Cost2') and spectrum usage gain (column 'Spec2') of flex-grid solutions with 400 Gbps line rates (scenario C) when compared with fixed-grid solution (scenarios A). The last line ('Average') presents the average gains among all 12 case studies.

TABLE III. COMPUTATIONAL RESULTS

\begin{tabular}{|l|c|c|c|r|r|r|}
\hline & CostA & CostB & CostC & SpecA & SpecB & SpecC \\
\hline 20L1 & 2380,8 & 2312,4 & 1860,0 & 60 & 54 & 52 \\
\hline 20L2 & 2111,2 & 2111,2 & 1820,8 & 72 & 72 & 70 \\
\hline 20H1 & 3655,6 & 3605,6 & 3138,8 & 88 & 93 & 77 \\
\hline 20H2 & 3898,0 & 3784,0 & 3159,2 & 80 & 79 & 65 \\
\hline 30L1 & 3187,6 & 3147,6 & 2606,0 & 108 & 108 & 96 \\
\hline 30L2 & 3569,2 & 3523,6 & 3198,8 & 128 & 124 & 118 \\
\hline 30H1 & 4872,0 & 4812,0 & 4248,4 & 106 & 112 & 112 \\
\hline 30H2 & 4461,6 & 4393,6 & 3795,6 & 134 & 130 & 88 \\
\hline 40L1 & 4474,8 & 4318,8 & 3866,8 & 104 & 96 & 96 \\
\hline 40L2 & 4721,2 & 4632,8 & 4026,4 & 138 & 134 & 112 \\
\hline 40H1 & 6734,0 & 6694,0 & 5594,8 & 154 & 154 & 112 \\
\hline 40H2 & 6204,8 & 6204,8 & 5186,4 & 140 & 140 & 128 \\
\hline
\end{tabular}

TABLE IV. COST AND SPECTRUM USAGE COMPARISON

\begin{tabular}{|c|r|r|r|r|}
\hline & \multicolumn{1}{|c|}{ Cost1 } & \multicolumn{1}{c|}{ Spec1 } & \multicolumn{1}{c|}{ Cost2 } & \multicolumn{1}{c|}{ Spec2 } \\
\hline $20 \mathrm{~L} 1$ & $-2.9 \%$ & $-10.0 \%$ & $-21.9 \%$ & $-13.3 \%$ \\
\hline $20 \mathrm{~L} 2$ & $0.0 \%$ & $0.0 \%$ & $-13.8 \%$ & $-2.8 \%$ \\
\hline $20 \mathrm{H} 1$ & $-1.4 \%$ & $5.7 \%$ & $-14.1 \%$ & $-12.5 \%$ \\
\hline $20 \mathrm{H} 2$ & $-2.9 \%$ & $-1.3 \%$ & $-19.0 \%$ & $-18.8 \%$ \\
\hline $30 \mathrm{~L} 1$ & $-1.3 \%$ & $0.0 \%$ & $-18.2 \%$ & $-11.1 \%$ \\
\hline $30 \mathrm{~L} 2$ & $-1.3 \%$ & $-3.1 \%$ & $-10.4 \%$ & $-7.8 \%$ \\
\hline $30 \mathrm{H} 1$ & $-1.2 \%$ & $5.7 \%$ & $-12.8 \%$ & $5.7 \%$ \\
\hline $30 \mathrm{H} 2$ & $-1.5 \%$ & $-3.0 \%$ & $-14.9 \%$ & $-34.3 \%$ \\
\hline $40 \mathrm{~L} 1$ & $-3.5 \%$ & $-7.7 \%$ & $-13.6 \%$ & $-7.7 \%$ \\
\hline $40 \mathrm{~L} 2$ & $-1.9 \%$ & $-2.9 \%$ & $-14.7 \%$ & $-18.8 \%$ \\
\hline $40 \mathrm{H} 1$ & $-0.6 \%$ & $0.0 \%$ & $-16.9 \%$ & $-27.3 \%$ \\
\hline $40 \mathrm{H} 2$ & $0.0 \%$ & $0.0 \%$ & $-16.4 \%$ & $-8.6 \%$ \\
\hline Average & $-1.5 \%$ & $-1.4 \%$ & $-15.6 \%$ & $-13.1 \%$ \\
\hline
\end{tabular}

These results show that both the cost and spectrum usage gains of flex-grid without introducing the $400 \mathrm{Gbps}$ line rate (OTU5 type lightpaths) are small, on average (1.5\% of cost gain and $1.4 \%$ of spectrum usage gain). Note that there are cases where there is no cost gain $(20 \mathrm{~L} 2$ and $40 \mathrm{H} 2)$ and others where a marginal cost gain is obtained at the expense of an higher spectrum usage (20H1 and $30 \mathrm{H} 1)$.

On the other hand, the solutions using flex-grid with 400 Gbps line rates exhibit significant gains, on average (15.6\% of cost gains and $13.1 \%$ of spectrum usage gains). Note that in most cases, solutions are significantly better on both parameters (cost and spectrum usage) and there is only one case $(30 \mathrm{H} 1)$ where a significant cost gain $(12.8 \%)$ is obtained at the expense of a spectrum usage which is $5.7 \%$ higher.

\section{CONCLUSIONS}

In this paper, we have addressed the design of optical networks aiming to optimize the design cost and the spectrum usage of the network. We have presented an exact method (i) to minimize the cost of the optical network (both fixed-grid and flex-grid) and (ii) to keep spectrum usage to a minimum. With such method, we have obtained the optimal solutions for case studies based on the 14-node NSFNET network. We have compared the results of these solutions. This comparison has revealed that the evolution of current fixed-grid optical networks to the future flex-grid technology is only worthy (in terms of network cost and spectrum usage) after the introduction of $400 \mathrm{Gbps}$ line rates provided by the OTU5 type of lightpaths. Before that, the evolution for the flex-grid technology does not result in significant gains for the network operator.

\section{ACKNOWLEDGMENT}

This work was partially supported through ONECI (Optimizing Next-generation Elastic Core Network Infrastructure) project, funded by the Fundação para Ciência e a Tecnologia - FCT (PTDC/EEITEL/3303/2012).

\section{REFERENCES}

[1] K. Christodoulopoulos, I. Tomkos, and E. Varvarigos, "Elastic bandwidth allocation in flexible OFDM-based optical networks," Journ. of Lightwave Tech., vol. 29, no. 9, pp. 1354-1366, May 2011.

[2] A. Morea, A. Chong, and O. Rival, "Impact of transparent network constraints on capacity gain of elastic channel spacing," Proc. OFC/NFOEC, JWA62, Los Angeles, March 2011

[3] O. Rival, and A. Morea, "Cost-efficiency of mixed 10-40-100 Gb/s networks and elastic optical networks," Proc. OFC/NFOEC, OTuI4, Los Angeles, March 2011.

[4] E. Palkopoulou, M. Angelou, D. Klonidis, K. Christodoulopoulos, A. Klekamp, F. Buchali, E. Varvarigos, and I. Tomkos, "Quantifying spectrum, cost, and energy efficiency in fixed-grid and flex-grid networks [invited]," Journ. of Optical Comm. and Netw., vol. 4, no. 11, pp. B42-B51, November 2012.

[5] K. Christodoulopoulos, P. Soumplis, and E. Varvarigos, "Planning flexible optical networks under physical layer constraints," Journ. of Optical Comm. and Netw., vol. 5, no. 11, pp. 1296-1312, November 2013.

[6] P. Monteiro, A. de Sousa, M. Ribeiro, T. Trota, G. Sahin, "Algorithms in the deployment of optical transport networks", Proc. ICTON, Cartagena, Spain, June 2013

[7] P. Wright, A. Lord, and L. Velasco, "The network capacity benefits of flexgrid," Proc. ONDM, Brest, April 2013.

[8] M. Tornatore, C. Rottondi, A. Morea, and G. Rizzelli, "Complexity and flexible grid networks [invited]," Proc. OFC, W3A1, San Francisco, March 2014

[9] F. Rambach, B. Konrad, L. Dembeck, U. Gebhard, M. Gunkel, M. Quagliotti, L. Serra, and V. Lopez, "A multilayer cost model for metro/core networks," Journ. of Optical Comm. and Net., vol. 5, no. 3, pp. 210-225, March 2013.

[10] [Online]. Available: http://www.nsfnet-legacy.org/about.php. 